\title{
The search for good relationships
}

\author{
Sophie Cook head of scholarly comment
}

The BMJ

Effective healthcare depends on complex relationships between patients, clinicians, regulators, managers, government, and industry. And these relationships work best where there is trust and transparency.

Failure to achieve these fundamentals creates tension, or a breakdown in relations, as seen recently during the negotiations over the UK junior doctors' contract and in the conviction and striking off of Hadiza Bawa-Garba.

This week we turn our attention to relationships between NHS trusts and the industry. An investigation by The BMJ and researchers from the University of Bath and Lund University in Sweden finds that these links are proliferating, encouraged by the UK government (doi:10.1136/bmj.11353). In 2016 and 2017 a quarter of acute care trusts in England were involved in joint projects with drug firms, on which the companies spent more than $£ 7.5 \mathrm{~m}(€ 8.7 \mathrm{~m} ; \$ 9.9 \mathrm{~m})$. Many of these projects involved new services to review medications or specific medical conditions but did not follow NHS rules on openness and transparency.

The industry says such relations can accelerate the spread of new treatments, but John Puntis, a consultant paediatrician and secretary of Keep Our NHS Public, reminds us that private companies' objectives differ fundamentally from those of NHS organisations. "It's not altruism; at the end of the day it's profit," he says. It can't be right that many of these agreements remain out of public sight.
Another strained relationship is between doctors and the GMC, which Lauren Morgan and colleagues describe as "in crisis" since the Bawa-Garba case, with doctors in fear of being individually blamed for making a mistake (doi:10.1136/bmj. 11037). These authors welcome the GMC's plans to consider human factors in fitness to practise cases: "A profession secure in the knowledge that it can trust its regulator to be just, and to understand the context of clinical error, is much more likely to be able to learn from mistakes and to lead to real sustainable improvements in patient safety."

The culture of fear among doctors may stem from how we talk about errors. Rammya Mathew argues that not all wrong calls are errors requiring a change in practice (doi:10.1136/bmj. 11234). "Appraisers should also be routinely asking us to reflect on the times when we got it wrong but concluded that changing our practice was not going to improve matters for anyone," she suggests.

Finally, even good relationships must come to an end. This week we bid farewell to BMJ Confidential. After five years of showcasing the innermost secrets of medical leaders and stalwarts around the globe, we end on a high note with our editor in chief, Fiona Godlee (doi:10.1136/bmj.11275). We thank everyone who has contributed to the series over the years and hope that their pearls of wisdom and candour continue to be an inspiration to others. 